\title{
Quark mass renormalization with non-exceptional momenta
}

\author{
Yasumichi Aoki* \\ RIKEN BNL Research Center, Brookhaven National Laboratory, Upton, NY 11973, USA \\ E-mail: yaoki@bnl.gov
}

\section{RBC and UKQCD collaborations}

Renormalization conditions imposed on quark bilinear vertex functions in the conventional RI/MOM scheme use exceptional momentum configurations. With practical values for the lattice cutoff, these vertex functions are contaminated with unwanted low energy physics (pion pole, zero modes, etc), which is a large source of systematic error. These effects can be reduced by using non-exceptional momenta. We discuss the quark mass renormalization with non-exceptional momenta using $2+1$ flavor domain wall fermions based on a recently proposed RI/SMOM scheme.

The XXVI International Symposium on Lattice Field Theory

July 14-19 2008

Williamsburg, Virginia, USA

\footnotetext{
*Speaker.
} 


\section{Introduction}

Quark masses are fundamental parameters in QCD. Recent lattice computations made it possible to calculate light quark masses up to strange consistently taking into account the light sea quark effects. The $2+1$ flavor domain wall fermion (DWF) calculation reported [1],

$$
\begin{aligned}
& m_{u d}^{\overline{\mathrm{MS}}}(2 \mathrm{GeV})=3.71(0.16)_{\text {stat }}(0.18)_{\text {syst }}(0.33)_{\text {ren MeV }}, \\
& m_{s}^{\overline{\mathrm{MS}}}(2 \mathrm{GeV})=107.3(4.4)_{\text {stat }}(4.9)_{\text {syst }}(9.7)_{\text {ren MeV }} .
\end{aligned}
$$

The first error is statistical. The second error is the systematic uncertainty in the determination of the bare quark mass in the lattice theory. It is dominated by the discretization error and will be significantly reduced when the ensemble on a finer lattice is analyzed: we are currently generating these configurations which will enable us to perform a continuum extrapolation. In this paper we discuss the reduction of the third error which arises in the renormalization of the mass. There are two dominant contributions to the error: (i) The non-perturbative renormalization of the mass in the RI/MOM scheme. We estimate the corresponding uncertainty to be about $7 \%$ due to the contamination by chiral symmetry breaking effects as explained below. (ii) The matching from the RI/MOM to the $\overline{\mathrm{MS}}$ scheme. The perturbative series for this matching is known to 3-loops [2, 3] but converges very poorly. The uncertainty is estimated to be about $6 \%$.

As was demonstrated in Ref. [П], the unwanted non-perturbative contaminations in RI/MOM scheme due to spontaneous chiral symmetry breaking could be reduced by changing the scheme to one in which no exceptional momenta are present. The argument stems from the Weinberg's theorem [5] on the behavior of the vertex function for large external momenta, where a set of external momenta which has zero partial sum is called exceptional.

In this article after the construction of an RI/MOM scheme with non-exceptional momenta for the quark bilinears is summarized, the method is applied to a data set used in the conventional RI/MOM renormalization with $2+1$ flavor DWFs [ $₫$ ]. The new results are compared with the conventional RI/MOM results.

\section{RI/SMOM scheme for quark mass}

\subsection{Conventional RI/MOM scheme}

We start briefly summarize the original RI/MOM scheme. A mass renormalization factor is completely fixed by introducing the two renormalization conditions on the quark propagator. For the conventional RI/MOM scheme, the conditions on the Landau-gauge propagator read

$$
\begin{aligned}
& \left.\frac{1}{12} \operatorname{Tr}\left[-i \frac{\partial}{\partial \not p} S_{R}^{-1}(p)\right]\right|_{p^{2}=\mu^{2}}=1, \\
& \lim _{m_{R} \rightarrow 0} \frac{1}{12 m_{R}} \operatorname{Tr}\left[S_{R}^{-1}(p)\right]_{p^{2}=\mu^{2}}=1,
\end{aligned}
$$

which are imposed at the mass-less point. The renormalized quark propagator and mass are related to the bare ones through

$$
S_{R}(p)=Z_{q}(\mu) S_{B}(p), \quad m_{R}=Z_{m}(\mu) m_{B} .
$$


Eq. (2.1) determines $Z_{q}^{\mathrm{MOM}}(\mu)$ which is needed for the Eq. (2.2), which in turn fixes $Z_{m}^{\mathrm{MOM}}(\mu)$. In the continuum theory, RI/MOM scheme wave function renormalization condition Eq. (2.1) can be rewritten in terms of the renormalization condition on the bare amputated Green function $\Pi$ of the vector current through the Ward-Takahashi identity as

$$
\left.\frac{1}{Z_{q}^{\mathrm{MOM}}} \frac{1}{48} \operatorname{Tr}\left[\gamma_{\mu} \Pi_{V_{\mu}}(p)\right]\right|_{p^{2}=\mu^{2}}=1,
$$

with $Z_{V}=1$. A similar relation applies for the axial vector vertex function, but with a contamination of a non-perturbative effect with $1 / p^{2}$ suppression [6],

$$
\left.\frac{1}{Z_{q}^{\mathrm{MOM}}}\left(\frac{1}{48} \operatorname{Tr}\left[\gamma_{5} \gamma_{\mu} \Pi_{A_{\mu}}(p)\right]+\frac{c_{N P}}{p^{2}}+\cdots\right)\right|_{p^{2}=\mu^{2}}=1,
$$

with $Z_{A}=1$. The momentum configuration must be stated to fix the renormalization condition. For these to give equivalent renormalization condition as Eq. (2.1), when the momentum $p$ comes in through one fermion line the same $p$ must go out via the other fermion line. This is an exceptional momentum configuration $\left(p_{1}+p_{2}=p-p=0\right)$. It is shown from the Weinberg's theorem [5] that the difference of the vector and axial vector vertex amplitude is $\sim 1 / p^{2}$ [4]. This is consistent with the existence of the $1 / p^{2}$ contamination term in Eq. (2.5), which Martinelli et al. derived through operator product expansion in Ref. [6].

On the lattice with DWFs, the use of vector or axial vector vertex function (Eqs. (2.4, 2.5) has an advantage over the quark propagator (Eq. (2.1) in calculating the quark wave function renormalization. The derivative with respect to momentum is not practical on the lattice as the momenta are quantized on the finite volume lattice. A similar scheme sometimes called as RI', in which Eq. (2.1) is replaced with

$$
\left.\frac{1}{12 p^{2}} \operatorname{Tr}\left[-i \not p S_{R}^{-1}(p)\right]\right|_{p^{2}=\mu^{2}}=1
$$

is free from the derivative. But, naive implementations of Eq. (2.6) on the lattice introduce the tree revel $(p a)^{2}$ error, which is sizable at the momentum range we use [7]. DWFs can utilize the conserved axial vector current and provide a precise estimate of $Z_{A}\left(=Z_{V}\right)$ [1]] of the local currents, which in turn allows one to use Eqs. (2.4), (2.5) with a correction of a $Z_{A}$ factor to get $Z_{q}^{\mathrm{MOM}}$.

By similar reasons, the mass renormalization should be calculated through bilinear operator renormalization using the relation $Z_{m}=1 / Z_{S}=1 / Z_{P}$. In principle, the scalar and pseudoscalar renormalization factors can be determined at large momenta by imposing the conditions

$$
\left.\frac{Z_{S}}{Z_{q}} \frac{1}{12} \operatorname{Tr}\left[\Pi_{S}(p)\right]\right|_{p^{2}=\mu^{2}}=1,\left.\quad \frac{Z_{P}}{Z_{q}} \frac{1}{12} \operatorname{Tr}\left[\gamma_{5} \Pi_{P}(p)\right]\right|_{p^{2}=\mu^{2}}=1 .
$$

At finite momenta however, $Z_{S}$ may differ from $Z_{P}$ due to spontaneous chiral symmetry breaking. In particular, for the exceptional momentum case, one needs to subtract the pion pole (for $P$ ) or double pole (for $S$ with quenching) to remove the divergence of $Z_{P}$ (and $Z_{S}$ quench) in the chiral limit. 


\subsection{RI/SMOM scheme}

Non-exceptional momenta do not have zero partial sum, which suppresses, in the Feynman diagram, the small momentum flow leading to non-perturbative contamination. Among various choices of non-exceptional momenta, we adopt the symmetric one $\mu^{2}=p_{1}^{2}=p_{2}^{2}=q^{2}$ where $q=$ $p_{1}-p_{2}$. This choice is convenient because only one invariant is involved $\left(2 p_{1} \cdot p_{2}\right.$ is also $\left.\mu^{2}\right)$. Here we briefly review the SMOM (symmetric MOM) scheme mass renormalization which makes use of the symmetric momentum configuration. The SMOM scheme is discussed in detail in Ref. [8]. We will demonstrate in the next section how the use of this renormalization scheme reduces the unwanted non-perturbative contaminations compared to the conventional MOM scheme.

Other than changing the momentum configuration the SMOM scheme follows the same steps as MOM scheme. The renormalization conditions are defined using trace conditions with specified projectors on the vertex functions $\Pi_{O}$. For the scalar and pseudoscalar operators, the same projection operators as MOM scheme $\frac{1}{12} 1, \frac{1}{12} \gamma_{5}$ as shown in Eq. (2.7) are used. The vector and axialvector operator will be used to calculate $Z_{q}$. Original MOM scheme uses $\frac{1}{48} \gamma_{\mu}, \frac{1}{48} \gamma_{5} \gamma_{\mu}$, by which one can relate the traced vertex function to $Z_{q}^{\mathrm{MOM}} \mathrm{Eq}$. (2.1). If we used these projection operators for the symmetric (non-exceptional) momenta, the resulting $Z_{q}$ would completely differ from that of MOM scheme (or RI' scheme). Instead, we adopt $\frac{1}{12 q^{2}} q_{q} q_{\mu}, \frac{1}{12 q^{2}} \gamma_{5} q_{q} q_{\mu}$. One can show that the use of these projection operators give $Z_{q}^{\mathrm{RI}}$ through the vector and axial vector Ward-Takahashi identities. The matching of $Z_{q}$ of RI' and $\overline{\mathrm{MS}}$ has been calculated to three loops [2, 3], which one can just use or can use for the check against the calculation with the vertex functions of vector and axialvector in the SMOM scheme. It is worth mentioning that RI' and RI/MOM $Z_{q}$ are same up to one loop. Thus, the resulting $Z_{q}$ from the vector and axialvector current of SMOM scheme should be close to that of original MOM scheme. The perturbative matching of quark mass from SMOM to $\overline{\mathrm{MS}}, m^{\overline{\mathrm{MS}}}(\mu)=C_{m}(\mathrm{SMOM} \rightarrow \overline{\mathrm{MS}}) \cdot m^{\mathrm{SMOM}}(\mu)$ has been calculated to one loop [8] as

$$
C_{m}(\mathrm{SMOM} \rightarrow \overline{\mathrm{MS}})=1-\frac{\alpha_{s}}{4 \pi} C_{F} \times(0.484-0.172 \xi)+O\left(\alpha_{s}^{2}\right),
$$

where $\xi$ is the gauge parameter. The same quantity for the original RI/MOM to $\overline{\mathrm{MS}}$ has much larger correction (both constant and linear coefficient of $\xi$ ):

$$
C_{m}(\mathrm{MOM} \rightarrow \overline{\mathrm{MS}})=1-\frac{\alpha_{s}}{4 \pi} C_{F} \times(4-\xi)+O\left(\alpha_{s}^{2}\right) .
$$

The one loop correction for the Landau gauge $(\xi=0)$ is $1.5 \%$ for SMOM and $12 \%$ for MOM at $\mu=2 \mathrm{GeV}$. The three loop correction is still large: $6 \%$ for MOM, which was taken as a conservative estimate of systematic error of perturbative matching [ [ 4 . The small correction of SMOM scheme is realized through cancellation of finite terms depending on the momentum structure. We have not understood if this is an universal property with SMOM scheme, which would persists beyond one loop.

\section{Numerical test of the RI/SMOM scheme}

We test the RI/SMOM scheme using the $N_{f}=2+1$ DWF data set [4] at $a^{-1} \simeq 1.7 \mathrm{GeV}$ on $16^{3} \times 32$ lattice with $L_{s}=16, M_{5}=1.8$. The quark propagators have been calculated with the point 


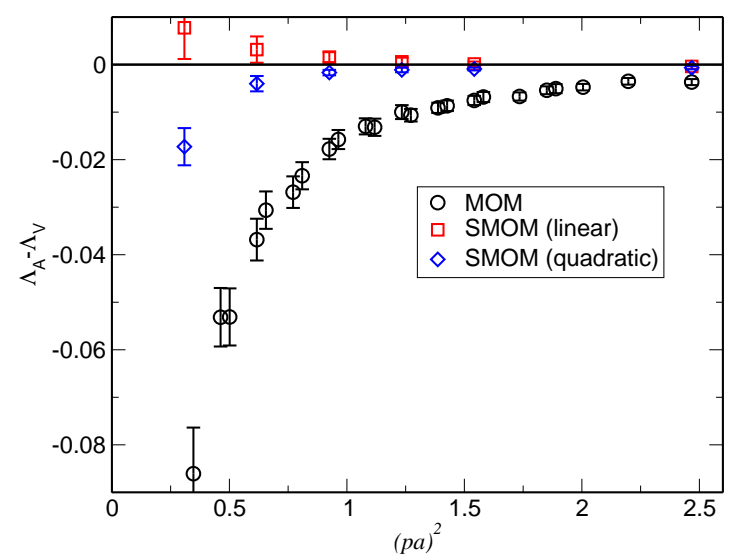

Figure 1: $\Lambda_{A}-\Lambda_{V}$ in the chiral limit as function of $p^{2}$ for MOM and SMOM scheme, where results with linear and quadratic chiral extrapolations in quark mass are shown.

source ${ }^{1}$.

Let us first look at the difference of the vector and axial vector vertex amplitude,

$$
\Lambda_{V}^{\mathrm{MOM}}=\frac{1}{48} \operatorname{Tr}\left[\gamma_{\mu} \Pi_{V_{\mu}}\right], \quad \quad \Lambda_{A}^{\mathrm{MOM}}=\frac{1}{48} \operatorname{Tr}\left[\gamma_{5} \gamma_{\mu} \Pi_{A_{\mu}}\right]
$$

for the exceptional momentum and for the symmetric (non-exceptional) momentum with

$$
\Lambda_{V}^{\mathrm{SMOM}}=\frac{1}{12 q^{2}} \operatorname{Tr}\left[\phi q_{\mu} \Pi_{V_{\mu}}\right], \quad \Lambda_{A}^{\mathrm{SMOM}}=\frac{1}{12 q^{2}} \operatorname{Tr}\left[\gamma_{5} \phi q_{\mu} \Pi_{A_{\mu}}\right] .
$$

Fig. 11 shows the differences in the chiral limit as functions of $p^{2}\left(=q^{2}\right)$. The original MOM scheme has non-zero difference due to the spontaneous chiral symmetry breaking. The decrease of the difference as momentum increases is due to the recovery of the symmetry and the size is up to $1 \%$ of the average $\left(\Lambda_{A}+\Lambda_{V}\right) / 2$ in the region of the momentum we use $(p a)^{2}>1.3$. The difference is much suppressed for the SMOM scheme. The linear chiral extrapolation gives results consistent with zero. The quadratic extrapolation gives non-zero value, but one order of magnitude smaller than MOM.

The scalar and pseudoscalar vertex amplitudes with MOM and SMOM scheme are shown in Fig. 2. A large difference between $P$ and $S$ is observed for the MOM scheme. Since $\Lambda_{P}$ diverges as $\sim 1 / m$ in the chiral limit due to the existence of pion pole $\langle\bar{\psi} \psi\rangle / m_{\pi}^{2}$ and $\Lambda_{S}$ stays finite, the difference becomes infinite. One should note that these quantities are identical to all order in perturbation theory. The symmetry is badly broken for the non-perturbative renormalization. On the other hand, $\Lambda_{P}$ and $\Lambda_{S}$ are consistent with each other for the SMOM case at larger momentum $(p a)^{2} \gtrsim 1$.

As our gauge ensembles have been sampled at single value of nearly physical strange mass, we have a systematic error from $m_{s} \neq 0$ even after the two-flavor unitary chiral extrapolation. This error in the MOM scheme, which turned out to be $7 \%$, was estimated from the response of the $\Lambda_{S}$ to the $u, d$ quark mass in Ref. [ $\llbracket$ ]. This error may represent the tolerance of this particular quantity

\footnotetext{
${ }^{1}$ The statistical error could be much improved if the volume source was used [9, 10, 11].
} 


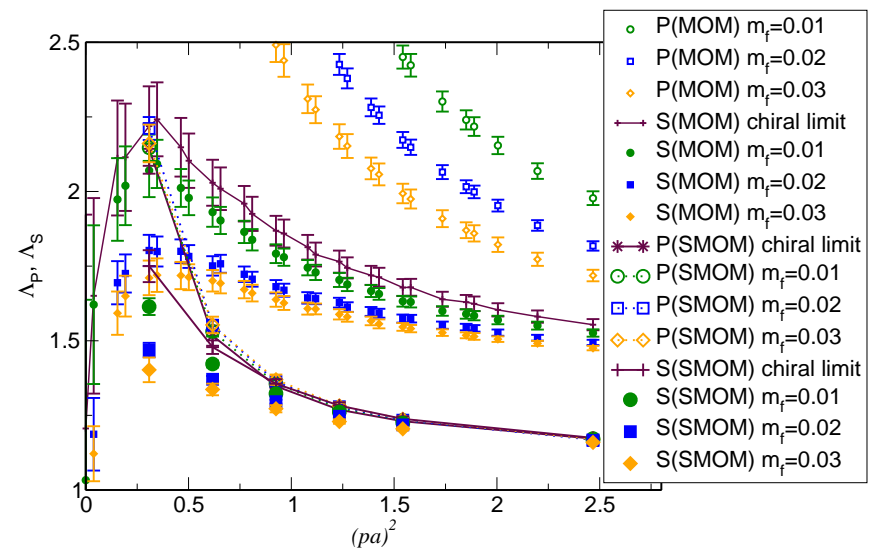

Figure 2: $\Lambda_{S}$ and $\Lambda_{P}$ for MOM and SMOM scheme. $m_{f}$ refers to the average $u, d$ mass. Points connected with the solid lines show the values in the two-flavor unitary chiral limit $\left(m_{f}+m_{\mathrm{res}} \rightarrow 0\right)$.

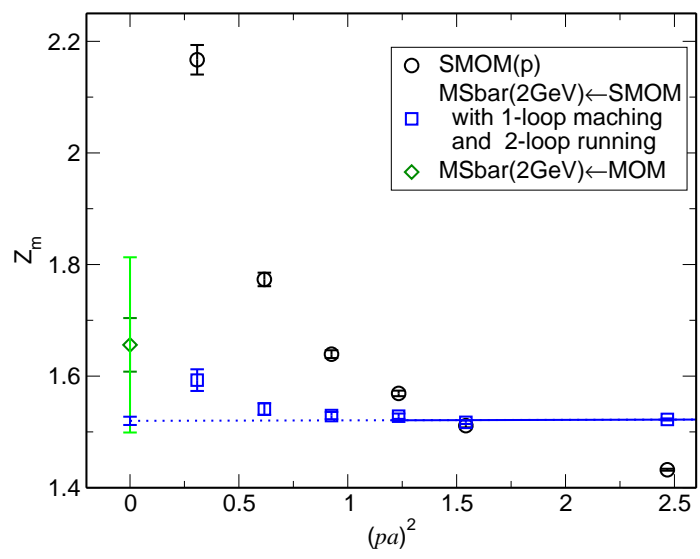

Figure 3: Preliminary results of $Z_{m}^{\mathrm{SMOM}}(p)$ as a function of renormalization scale $p$ and $Z_{m}^{\overline{\mathrm{MS}}}(\mu=2 \mathrm{GeV})$ as a function of SMOM $\rightarrow \overline{\mathrm{MS}}$ matching scale $p$. The extrapolation $(p a)^{2} \rightarrow 0$ is shown with statistical error only, which is consistent with the $Z_{m}^{\overline{\mathrm{MS}}}(\mu=2 \mathrm{GeV})$ through MOM scheme with the large systematic error.

to the emergence of the low energy scale $\left(\sim \Lambda_{Q C D}\right)$. So even if the $m_{s} \rightarrow 0$ limit was performed, error of similar size would remain due to the non-perturbative effect whose energy scale is about the same. Now, in the SMOM case, as shown in the figure, mass dependence is greatly reduced. If we adopt the same method, the systematic error of SMOM is about $3 \%$ for the scalar or negligible (comparable to the statistical error) for the pseudoscalar.

Black symbols in Fig. 3 show preliminary results of $Z_{m}=\left(\Lambda_{S}+\Lambda_{P}\right) /\left\{Z_{A}\left(\Lambda_{A}+\Lambda_{V}\right)\right\}$ with SMOM scheme (in the chiral limit) as a function of renormalization scale $p$, where the $Z_{A}$ [1] estimated form hadronic two point functions is used. Matching to $\overline{\mathrm{MS}}$ with Eq. (2.8) and running to $\mu=2 \mathrm{GeV}$ with the two-loop anomalous dimension, one obtains the blue symbols as a function of matching scale $p$. The extrapolation $(p a)^{2} \rightarrow 0$ using the points $(p a)^{2} \gtrsim 1.2$ gives a consistent result with the same quantity but through original MOM scheme [円] with 3-loop matching and 4-loop running. 


\section{Conclusion}

The RI/SMOM scheme, constructed in the framework of the conventional RI/MOM scheme with the use of non-exceptional momenta, works very well for reducing non-perturbative contamination for the quark mass renormalization. The systematic error is reduced to $3 \%$ level for $Z_{m}$, while it was 7\% for the original MOM scheme. This shows the success of the SMOM scheme which was designed to reduce the unwanted non-perturbative contamination. Another systematic error is from truncation in the perturbative matching to $\overline{\mathrm{MS}}$. If we estimate the systematic error for the SMOM scheme from the size of $O\left(\alpha_{s}\right)$ at our typical momentum size $\mu=2 \mathrm{GeV}$, it is $1.5 \%$, which is much smaller than $6 \%$ at $O\left(\alpha_{s}^{3}\right)$ for the MOM scheme. Further discussions are needed for the better understanding of the systematic error of the perturbative matching.

The first non-trivial test of the SMOM scheme was successful. Application to other bilinear operators such as tensors would be straightforward. Similar scheme can be constructed for fourquark operators for $K^{0}-\overline{K^{0}}$ mixing in the standard model and beyond, and for $K \rightarrow \pi \pi$ decays.

We thank Christian Sturm for collaborating on the RI/SMOM renormalization. Presented numerical data are obtained through reanalyzing the published data computed on the QCDOC machines at RIKEN BNL Research Center, Columbia University and University of Edinburgh.

\section{References}

[1] RBC-UKQCD Collaboration, C. Allton et. al., Physical Results from 2+1 Flavor Domain Wall QCD and SU(2) Chiral Perturbation Theory, Phys. Rev. D78 (2008) 114509, arXiv: 0804.0473 ].

[2] K. G. Chetyrkin and A. Retey, Renormalization and running of quark mass and field in the regularization invariant and ms-bar schemes at three and four loops, Nucl. Phys. $\mathbf{B 5 8 3}$ (2000) 3-34, [hep-ph/9910332].

[3] J. A. Gracey, Three loop anomalous dimension of non-singlet quark currents in the ri' scheme, Nucl. Phys. B662 (2003) 247-278, hep-ph/0304113].

[4] Y. Aoki et. al., Non-perturbative renormalization of quark bilinear operators and $B_{K}$ using domain wall fermions, Phys. Rev. D78 (2008) 054510, [arXiv: 0712.1061].

[5] S. Weinberg, High-energy behavior in quantum field theory, Phys. Rev. 118 (1960) 838-849.

[6] G. Martinelli et. al., A General method for nonperturbative renormalization of lattice operators, Nucl. Phys. B445 (1995) 81-108, [hep-lat/9411010].

[7] T. Blum et. al., Non-perturbative renormalisation of domain wall fermions: Quark bilinears, Phys. Rev. D66 (2002) 014504, hep-lat/0102005].

[8] C. Sturm et. al., Renormalization of quark bilinear operators in a MOM- scheme with a non-exceptional subtraction point, arXiv:0901.2599.

[9] RBC and UKQCD Collaboration, P. A. Boyle et. al., Parton distribution amplitudes and non-perturbative renormalisation, PoS(LATTICE 2008)165 (2008).

[10] RBC and UKQCD Collaboration, C. Kelly, Scaling of $b_{k}$ for $2+1$ flavour domain wall fermions from $24^{3}$ and $32^{3} \times 64$ lattices, PoS(LATTICE 2008)270 (2008).

[11] RBC and UKQCD Collaboration, J. Wennekers, Neutral kaon mixing beyond the standard model from 2+1 flavour domain wall qcd, PoS(LATTICE 2008)269 (2008). 